\title{
LINEAR MAPS PRESERVING DRAZIN INVERSES OF MATRICES OVER LOCAL RINGS
}

\author{
TUGCE PEKACAR CALCI, HUANYIN CHEN, SAIT HALICIOGLU, AND GUO SHILE
}

Abstract. Let $R$ be a local ring and suppose that there exists $a \in F^{*}$ such that $a^{6} \neq 1$; also let $T: M_{n}(R) \rightarrow M_{m}(R)$ be a linear map preserving Drazin inverses. Then we prove that $T=0$ or $n=m$ and $T$ preserves idempotents. We thereby determine the form of linear maps from $M_{n}(R)$ to $M_{m}(R)$ preserving Drazin inverses of matrices.

\section{INTRODUCTION}

Let $R$ be a commutative ring with an identity. $M_{n}(R)$ denotes the $n \times n$ matrix algebra over $R$ and $G L_{n}(R)$ stands for the general linear group of $M_{n}(R)$ for a positive integer $n$. A matrix $A \in M_{n}(R)$ has Drazin inverse if there exists $B \in M_{n}(R)$ such that

$$
B=B A B, \quad A B=B A, \quad A^{k}=A^{k+1} B \quad \text { for some } k \in \mathbb{N} .
$$

The preceding $B$ is unique if it exists; we denote it by $A^{D}$. The Drazin inverse plays an important role in matrix and operator theory (see [7, 12, 13, 15, 14]). We say that a linear map $T: M_{n}(R) \rightarrow M_{m}(R)$ preserves Drazin inverses of matrices if the condition " $A \in M_{n}(R)$ has Drazin inverse" implies that $T(A) \in M_{m}(R)$ has Drazin inverse and $T(A)^{D}=T\left(A^{D}\right)$. Linear maps preserving generalized inverses of matrices are extensively studied by many authors, e.g., [1, 2, 3, 4, 6, 8, 9, 10, 11, 12 .

Recall that a ring $R$ is local if $R$ has exactly one maximal ideal $M$. The $\operatorname{ring} R / M$ is called the residue field of $R$; we denote it by $F$. It is well known that a ring $R$ is local if and only if for any $x \in R$, either $x$ or $1-x$ is invertible. Clearly, every field is a local ring. The purpose of this paper is to further explore the linear maps preserving Drazin inverses of matrices over local rings. Let $T: M_{n}(R) \rightarrow M_{m}(R)$ be a linear map preserving Drazin inverses. If $a^{6} \neq 1$ for some $a \in F^{*}$, we prove that $T=0$ or $n=m$ and $T$ preserves idempotents. That is, the preserving of Drazin

2020 Mathematics Subject Classification. 15A86, 15A09, 13 H99.

Key words and phrases. Linear map; Drazin inverse; local ring.

The first and the third authors are supported by The Scientific and Technological Research Council of Turkey, TUBITAK (Grant TUBITAK-116F435). 
inverses can be reduced to the case of idempotents. We thereby determine the form of linear maps from $M_{n}(R)$ to $M_{m}(R)$ preserving Drazin inverses of matrices.

In what follows, $\mathbb{Z}$ and $\mathbb{Z}_{n}$ denote respectively the ring of integers and the ring of integers modulo $n$ for some positive integer $n$. We write $J(R)$ and $U(R)$ for the Jacobson radical of $R$ and the set of all invertible elements of $R$, respectively.

Throughout the paper, $R$ is a commutative local ring with the residue field $F$. Also, $F^{*}$ denotes the group of all nonzero elements in the field $F$. Moreover, $E_{i j}$ denotes the matrices with 1 in the $(i, j)$-entry and 0 elsewhere, for any $i, j \in[1, n]$.

\section{MAin RESUlts}

Let $R$ be a local ring with the residue field $F$, and let $T$ be a linear map from $M_{n}(R)$ to $M_{m}(R)$, with $m, n>1$. The aim of this section is to investigate the linear maps preserving Drazin inverses of matrices for such a local ring $R$.

Lemma 2.1. Let $R$ be a local ring and suppose that there exists $a \in F^{*}$ such that $a^{6} \neq 1$. Let $T$ be a linear map from $M_{n}(R)$ to $M_{m}(R)$. If $T$ preserves Drazin inverses of matrices and $T\left(E_{i i}\right)=0$ for some $i \in[1, n]$, then $T=0$.

Proof. We claim that $|F| \geq 4$. If not, $\left|F^{*}\right|<3$, and so either $x^{3}=1$ or $x^{3}=-1$ for any $x \in F^{*}$. This shows that $x^{6}=1$, a contradiction. We may assume $T\left(E_{11}\right)=0$. Since $|F| \geq 4$, we find some $\bar{a} \notin\{\overline{0}, \overline{1}, \overline{-1}\}$. Then $a \in U(R)$. Let $x \in U(R)$. Then $\left(E_{11}+x E_{1 j}\right)^{D}=E_{11}+x E_{1 j}$ for any $j \in[2, n]$. Hence,

$$
T\left(E_{11}+x E_{1 j}\right)^{3}=T\left(E_{11}+x E_{1 j}\right)
$$

and so $x^{3} T\left(E_{1 j}\right)^{3}=x T\left(E_{1 j}\right)$. In particular choose $x=1$ and $x=a$; we see that

$$
T\left(E_{1 j}\right)^{3}=T\left(E_{1 j}\right), \quad a^{2} T\left(E_{1 j}\right)^{3}=T\left(E_{1 j}\right) .
$$

Thus, we have $(a-1)(a+1) T\left(E_{1 j}\right)=0$. Clearly, $a-1, a+1 \in U(R)$; hence, $T\left(E_{1 j}\right)=0$. Likewise, $T\left(E_{j 1}\right)=0$ for any $j \in[2, n]$. Set $X=E_{11}+E_{1 j}+E_{j 1}$ and $A=E_{1 j}+E_{j 1}-E_{j j}$, where $j \in[2, n]$. Then $T(X)=0$ and $A^{D}=X$. Then $T(A)^{k}=T(A)^{k+1} T(X)=0$, and so $(-1)^{k} T\left(E_{j j}\right)=0$. Hence $T\left(E_{j j}\right)=0$. Therefore $T=0$, as asserted.

Lemma 2.2. Let $R$ be a local ring and suppose that there exists $a \in F^{*}$ such that $a^{6} \neq 1$. Let $T$ be a linear map from $M_{n}(R)$ to $M_{m}(R)$. If $T$ preserves Drazin inverses of matrices, then

$$
T\left(E_{i i}\right) T\left(E_{j j}\right)=T\left(E_{j j}\right) T\left(E_{i i}\right)=0
$$

for any distinct $i, j \in[1, n]$.

Proof. Since $a^{6} \neq 1$ for some $a \in F^{*}$, from the above discussion we easily see that $|F| \geq 4$, and so we can find some $\bar{a} \notin\{\overline{0}, \overline{1}, \overline{-1}\}$. Then $a \in U(R)$. Let $A=E_{i i}+a^{-1} E_{j j}, X=E_{i i}+a E_{j j}$ for distinct $i, j \in[1, n]$. Then $X=A^{D}$. Hence we have

$$
T\left(E_{i i}+a E_{j j}\right) T\left(E_{i i}+a^{-1} E_{j j}\right)=T\left(E_{i i}+a^{-1} E_{j j}\right) T\left(E_{i i}+a E_{j j}\right) .
$$


It follows that

$$
\left(a^{-1}-a\right)\left(T\left(E_{i i}\right) T\left(E_{j j}\right)-T\left(E_{j j}\right) T\left(E_{i i}\right)\right)=0 .
$$

As $a^{2}-1 \in U(R)$, we see that $T\left(E_{i i}\right) T\left(E_{j j}\right)=T\left(E_{j j}\right) T\left(E_{i i}\right)$.

Let $i, j \in[1, n]$ be distinct. By hypothesis, there exists some $a \in U(R)$ such that $\bar{a}^{6} \neq \overline{1}$ in $F$. Set $Y=E_{i i}+a E_{j j}$ and $B=E_{i i}+a^{-1} E_{j j}$. Then $Y=B^{D}$. Hence

$$
T(Y)=T\left(B^{D}\right)=T(B)^{D}=\left(T(B)^{D}\right)^{2} T(B)=T(Y)^{2} T(B) .
$$

It follows that

$$
\begin{aligned}
T\left(E_{i i}\right)+a T\left(E_{j j}\right)= & \left(T\left(E_{i i}\right)+a T\left(E_{j j}\right)\right)^{2}\left(T\left(E_{i i}\right)+a^{-1} T\left(E_{j j}\right)\right) \\
= & T\left(E_{i i}\right)^{3}+\left(a^{-1}+2 a\right) T\left(E_{i i}\right)^{2} T\left(E_{j j}\right) \\
& +\left(2+a^{2}\right) T\left(E_{i i}\right) T\left(E_{j j}\right)^{2}+a T\left(E_{j j}\right)^{3} .
\end{aligned}
$$

Since $E_{i i}$ is an idempotent, $E_{i i}^{D}=E_{i i}$ and so $T\left(E_{i i}\right)^{D}=T\left(E_{i i}^{D}\right)=T\left(E_{i i}\right)$. Hence it is easy to see that

$$
T\left(E_{i i}\right)=T\left(E_{i i}\right)^{3} .
$$

Similarly, $T\left(E_{j j}\right)=T\left(E_{j j}\right)^{3}$. Hence,

$$
\left(a^{-1}+2 a\right) T\left(E_{i i}\right)^{2} T\left(E_{j j}\right)+\left(2+a^{2}\right) T\left(E_{i i}\right) T\left(E_{j j}\right)^{2}=0 .
$$

That is,

$$
\left(1+2 a^{2}\right) T\left(E_{i i}\right)^{2} T\left(E_{j j}\right)+\left(2 a+a^{3}\right) T\left(E_{i i}\right) T\left(E_{j j}\right)^{2}=0 .
$$

Since $T\left(E_{i i}\right)=T\left(E_{i i}\right)^{3}$ and $T\left(E_{j j}\right)=T\left(E_{j j}\right)^{3}$, we derive

$$
\left(2 a+a^{3}\right) T\left(E_{i i}\right)^{2} T\left(E_{j j}\right)+\left(1+2 a^{2}\right) T\left(E_{i i}\right) T\left(E_{j j}\right)^{2}=0 .
$$

Combining (2.1) and (2.2), we have

$$
(a-1)\left(a^{2}-a+1\right)\left(T\left(E_{i i}\right)^{2} T\left(E_{j j}\right)-T\left(E_{i i}\right) T\left(E_{j j}\right)^{2}\right)=0 .
$$

Clearly,

$$
\overline{(a-1)\left(a^{2}-a+1\right)(a+1)\left(a^{2}+a+1\right)}=\overline{a^{6}-1} \neq \overline{0}
$$

in $F$; hence,

$$
(a-1)\left(a^{2}-a+1\right)(a+1)\left(a^{2}+a+1\right) \in U(R) .
$$

Therefore we get

$$
T\left(E_{i i}\right)^{2} T\left(E_{j j}\right)=T\left(E_{i i}\right) T\left(E_{j j}\right)^{2} .
$$

It follows by $(2.2)$ that

$$
(a+1)\left(a^{2}+a+1\right) T\left(E_{i i}\right)^{2} T\left(E_{j j}\right)=0,
$$

and then $T\left(E_{i i}\right)^{2} T\left(E_{j j}\right)=0$. Consequently, we have

$$
T\left(E_{i i}\right) T\left(E_{j j}\right)=T\left(E_{i i}\right)^{3} T\left(E_{j j}\right)=0 .
$$

This completes the proof.

Lemma 2.3. Let $R$ be a local ring, and let $A^{3}=A \in M_{n}(R)$. Then there exists $P \in G L_{n}(R)$ such that $P A P^{-1}=\operatorname{diag}\left(A_{1}, 0_{n-r}\right)$, where $A_{1}^{2}=I_{r}$ for some $r \in$ $[0, n]$. 
Proof. Clearly, $A$ is regular. Since $R$ is a local ring, it follows from [5, Theorem 7.3.2] that there exist $P, Q \in G L_{n}(R)$ such that

$$
P A Q=\operatorname{diag}\left(I_{r}, d_{1}, \ldots, d_{n-r}\right),
$$

where $d_{i} \in J(R)$ for $i \in[1, n-r]$. Since $A$ is regular, so is $P A Q$, and then each $d_{i} \in R$ is regular. Write $d_{i}=d_{i} x_{i} d_{i}$ for some $x_{i} \in R$. Then $d_{i}\left(1-x_{i} d_{i}\right)=0$. As $d_{i} \in J(R)$, we see that $1-x_{i} d_{i} \in U(R)$; hence, $d_{i}=0$. Therefore $P A Q=$ $\operatorname{diag}\left(I_{r}, 0_{n-r}\right)$. We have

$$
P A P^{-1}=\operatorname{diag}\left(I_{r}, 0_{n-r}\right) Q^{-1} P^{-1}=\left(\begin{array}{cc}
A_{1} & A_{2} \\
0 & 0
\end{array}\right) .
$$

Since $A^{3}=A$, we get

$$
A_{1}^{2}\left(A_{1}, A_{2}\right)=\left(A_{1}, A_{2}\right) .
$$

Choose $Y=P Q$. Then we have $\left(A_{1}, A_{2}\right) Y=I_{r}$, and so $A_{1}^{2}=I_{r}$. Hence,

$$
\left(\begin{array}{cc}
I_{r} & A_{1}^{-1} A_{2} \\
0 & I_{n-r}
\end{array}\right) P A P^{-1}\left(\begin{array}{cc}
I_{r} & -A_{1}^{-1} A_{2} \\
0 & I_{n-r}
\end{array}\right)=\left(\begin{array}{cc}
A_{1} & 0 \\
0 & 0
\end{array}\right) \text {. }
$$

This completes the proof.

Lemma 2.4. Let $R$ be a local ring and suppose that there exists $a \in F^{*}$ such that $a^{6} \neq 1$. Let $T$ be a linear map from $M_{n}(R)$ to $M_{m}(R), n \geq m$. If $T$ preserves Drazin inverses of matrices, then $T=0$ or $n=m$ and $T\left(I_{n}\right)=I_{n}$.

Proof. If $T\left(E_{i i}\right)=0$ for some $i \in[1, n]$, it follows by Lemma 2.1 that $T=0$. Next, we assume that $T\left(E_{i i}\right) \neq 0$ for all $i \in[1, n]$.

Since $E_{11}^{3}=E_{11}$, by virtue of Lemma 2.3 there exists $P_{1} \in G L_{m}(R)$ such that

$$
T\left(E_{11}\right)=P_{1}\left(\begin{array}{cc}
A_{1} & 0 \\
0 & 0
\end{array}\right) P_{1}^{-1}
$$

where $A_{1}^{2}=I_{r_{1}}$ for some $r_{1} \in[0, m]$. Let

$$
T\left(E_{22}\right)=P_{1}\left(\begin{array}{ll}
X_{11} & X_{12} \\
X_{21} & X_{22}
\end{array}\right) P_{1}^{-1}
$$

where $X_{11} \in M_{r_{1}}(R)$. In view of Lemma 2.2 we have

$$
T\left(E_{11}\right) T\left(E_{22}\right)=T\left(E_{22}\right) T\left(E_{11}\right)=0,
$$

and so $X_{12}=0, X_{21}=0$ and $X_{11}=0$. Thus,

$$
T\left(E_{22}\right)=P_{1}\left(\begin{array}{cc}
0 & 0 \\
0 & X_{22}
\end{array}\right) P_{1}^{-1},
$$

where $X_{22}^{3}=X_{22}$. By using Lemma 2.3 again, there exists $Q_{1} \in G L_{m-r_{1}}(R)$ such that

$$
X_{22}=Q_{1}\left(\begin{array}{cc}
A_{2} & 0 \\
0 & 0
\end{array}\right) Q_{1}^{-1}
$$


where $A_{2}^{2}=I_{r_{2}}$ for some $r_{2} \in\left[0, m-r_{1}\right]$. It follows that

$$
T\left(E_{22}\right)=P_{1}\left(\begin{array}{cc}
I_{r_{1}} & 0 \\
0 & Q_{1}
\end{array}\right)\left(\begin{array}{ccc}
0 & 0 & 0 \\
0 & A_{2} & 0 \\
0 & 0 & 0
\end{array}\right)\left(\begin{array}{cc}
I_{r_{1}} & 0 \\
0 & Q_{1}^{-1}
\end{array}\right) P_{1}^{-1} .
$$

Set $P_{2}=P_{1}\left(\begin{array}{cc}I_{r_{1}} & 0 \\ 0 & Q_{1}\end{array}\right)$. Then

$$
T\left(E_{22}\right)=P_{2}\left(0 \oplus A_{2} \oplus 0\right) P_{2}^{-1} .
$$

Moreover,

$$
T\left(E_{11}\right)=P_{2}\left(A_{1} \oplus 0 \oplus 0\right) P_{2}^{-1} .
$$

By iteration of this process, we have

$$
T\left(E_{i i}\right)=P\left(0 \oplus \cdots \oplus A_{i} \oplus \cdots \oplus 0\right) P^{-1},
$$

where $A_{i}^{2}=I_{r_{i}}$. Clearly, $A_{i} \neq 0$, and so

$$
T\left(I_{n}\right)=T\left(E_{11}+\cdots+E_{n n}\right)=P\left(A_{1} \oplus \cdots \oplus A_{n}\right) P^{-1},
$$

where $A_{i}^{2}=I_{r_{i}}$. As $n \geq m$, we see that $n=m$, and so $A_{i}=1$ for any $i \in[1, n]$. Therefore $T\left(I_{n}\right)=I_{n}$, as asserted.

Lemma 2.5. Let $R$ be a local ring and suppose that there exists $a \in F^{*}$ such that $a^{6} \neq 1$. Let $T$ be a linear map from $M_{n}(R)$ to $M_{n}(R)$. If $T$ preserves Drazin inverses of matrices, then $T\left(E_{i i}\right)=T\left(E_{i i}\right)^{2}$ for all $i \in[1, n]$.

Proof. If $T=0$, then the result holds. We may assume that $T \neq 0$. In light of Lemma 2.4 $T\left(I_{n}\right)=I_{n}$. As in the proof of Lemma 2.1. since $|F| \geq 4$, we can find some $\bar{x} \notin\{\overline{0}, \overline{1}, \overline{2}\}$. Clearly, we have

$$
\left(I_{n}+\left(x^{-1}-1\right) E_{i i}\right)^{D}=I_{n}+(x-1) E_{i i},
$$

and so

$$
T\left(I_{n}+(x-1) E_{i i}\right) T\left(I_{n}+\left(x^{-1}-1\right) E_{i i}\right) T\left(I_{n}+(x-1) E_{i i}\right)=T\left(I_{n}+(x-1) E_{i i}\right) .
$$

Set $A=T\left(E_{i i}\right)$. Then,

$$
\left(I_{n}+(x-1) A\right)\left(I_{n}+\left(x^{-1}-1\right) A\right)\left(I_{n}+(x-1) A\right)=I_{n}+(x-1) A .
$$

Hence,

$$
\begin{aligned}
\left(I_{n}+(x-1) A\right)\left(I_{n}(x-1+1)-(x-1) A\right)\left(I_{n}+(x-1) A\right) & \\
& =I_{n}(x-1+1)+(x-1+1)(x-1) A .
\end{aligned}
$$

This shows that

$$
\begin{aligned}
\left(I_{n}+(x-1) A\right)\left(I_{n}+(x-1)\left(I_{n}-A\right)\right)\left(I_{n}\right. & +(x-1) A) \\
& =I_{n}+(x-1)\left(I_{n}+A\right)+(x-1)^{2} A
\end{aligned}
$$

and so

$$
(x-1)^{2}\left(A-A^{2}\right)\left(I_{n}+(x-1) A\right)=0 .
$$


Since $A^{3}=A$, we have

$$
(x-1)^{2}(2-x)\left(A-A^{2}\right)=0 .
$$

As $x-1, x-2 \in U(R)$, we see that $A^{2}=A$, as asserted.

We have accumulated all the information necessary to prove the following result.

Theorem 2.6. Let $R$ be a local ring and suppose that there exists $a \in F^{*}$ such that $a^{6} \neq 1$. Let $T$ be a linear map from $M_{n}(R)$ to $M_{m}(R), n \geq m$. If $T$ preserves Drazin inverses of matrices, then $T=0$ or $n=m$ and $T$ preserves idempotents.

Proof. Suppose that $T \neq 0$. In view of Lemma 2.4 $n=m$ and $T\left(I_{n}\right)=I_{n}$. Let $M^{2}=M \in M_{n}(R)$. Then there exists $Q \in G L_{n}(R)$ such that $M=Q\left(I_{r} \oplus 0\right) Q^{-1}$. Let $T_{1}(X)=T\left(Q X Q^{-1}\right)$. Then $T_{1}$ is a linear map from $M_{n}(R)$ to $M_{n}(R)$ and it preserves Drazin inverses of matrices with $T_{1}\left(I_{n}\right)=I_{n}$. By Lemma 2.2

$$
T_{1}\left(E_{i i}\right) T_{1}\left(E_{j j}\right)=T_{1}\left(E_{j j}\right) T_{1}\left(E_{i i}\right)=0
$$

for any distinct $i, j \in[1, n]$. By Lemma 2.5. $T_{1}\left(E_{i i}\right)=T_{1}\left(E_{i i}\right)^{2}$ for any $i \in[1, n]$. Therefore,

$$
\begin{aligned}
T(M) & =T\left(Q\left(I_{r} \oplus 0\right) Q^{-1}\right) \\
& =T_{1}\left(I_{r} \oplus 0\right) \\
& =\sum_{i=1}^{r} T_{1}\left(E_{i i}\right) \\
& =\sum_{i=1}^{r} T_{1}\left(E_{i i}\right)^{2} \\
& =\left(\sum_{i=1}^{r} T_{1}\left(E_{i i}\right)\right)^{2} \\
& =\left(T_{1}\left(I_{r} \oplus 0\right)\right)^{2} \\
& =T(M)^{2},
\end{aligned}
$$

as asserted.

Note that the trivial map $T=0$ preserves Drazin inverses of matrices. For the nonzero case, we have the following.

Corollary 2.7. Let $R$ be a local ring with $2,3,7 \in U(R)$, and let $T$ be a nonzero linear map from $M_{n}(R)$ to $M_{m}(R)$. Then $T$ preserves Drazin inverses of matrices if and only if $n=m$ and either there exists $P \in G L_{n}(R)$ such that $T(A)=P A P^{-1}$ or there exists $P \in G L_{n}(R)$ such that $T(A)=P A^{t} P^{-1}$.

Proof. If $a^{6}=1$ for all $x \in F^{*}$, then $2^{6}=1$. Hence, $3^{2} \times 7=0$, a contradiction. Therefore we can find some $a \in F^{*}$ such that $a^{6} \neq 1$. In view of Theorem 2.6. $T$ preserves idempotents. Therefore we complete the proof by [3, Theorem].

We now construct a ring to illustrate the preceding result. 
Example 2.8. Let $\mathbb{Z}_{(5)}=\left\{\frac{p}{q} \mid p, q \in \mathbb{Z},(p, q)=1\right.$ and $\left.5 \nmid q\right\}$. Then $\mathbb{Z}_{(5)}$ is a local ring with $2,3,7 \in U\left(\mathbb{Z}_{(5)}\right)$. Let $T$ be a linear map from $M_{n}\left(\mathbb{Z}_{(5)}\right)$ to $M_{m}\left(\mathbb{Z}_{(5)}\right)$. Then $T$ preserves Drazin inverses of matrices if and only if $T$ has the forms as in Corollary 2.7

Proof. Clearly, $J\left(\mathbb{Z}_{(5)}\right)=5 \mathbb{Z}_{(5)}$, and so $\mathbb{Z}_{(5)} / J\left(\mathbb{Z}_{(5)}\right) \cong 5 \mathbb{Z}$. Therefore we are through by Corollary 2.7 .

The condition " $a^{6} \neq 1$ for some $a \in F^{*}$ " in Theorem 2.6 is not superfluous, as the following shows.

Example 2.9. Let $T: M_{2}\left(\mathbb{Z}_{3}\right) \rightarrow M_{2}\left(\mathbb{Z}_{3}\right)$ be the linear map given by

$$
\left(\begin{array}{ll}
a & b \\
c & d
\end{array}\right) \mapsto\left(\begin{array}{cc}
-a & -c \\
-b & -d
\end{array}\right) \text {. }
$$

Then $T$ preserves Drazin inverses of matrices, but $T$ does not preserve idempotents.

Proof. Clearly, $\mathbb{Z}_{3}$ is local. Since $T(A)=-A^{t}$ for any $A \in M_{2}\left(\mathbb{Z}_{3}\right)$, we easily check that $T$ preserves Drazin inverses of matrices. But

$$
T\left(\left(\begin{array}{ll}
1 & 0 \\
1 & 0
\end{array}\right)\right)=\left(\begin{array}{cc}
-1 & -1 \\
0 & 0
\end{array}\right)
$$

which is not an idempotent. Therefore $T$ does not preserve idempotents. Observe that in this case, $a^{6}=1$ for all $a \in \mathbb{Z}_{3}^{*}$.

\section{ACKNOWLEDGEMENT}

The authors would like to thank the referee for his/her careful reading of the manuscript.

\section{REFERENCES}

[1] N. Boudi and M. Mbekhta, Additive maps preserving strongly generalized inverses, J. Operator Theory 64 (2010), no. 1, 117-130. MR 2669430

[2] C. Bu, Linear maps preserving Drazin inverses of matrices over fields, Linear Algebra Appl. 396 (2005), 159-173. MR 2112204

[3] C. G. Cao, Linear maps preserving idempotence of matrix modules over certain rings. (Chinese. English, Chinese summary) Heilongjiang Daxue Ziran Kexue Xuebao 16 (1999), no. 1, 1-4. MR 1725451

[4] C.-G. Cao and X. Zhang, Linear preservers between matrix modules over connected commutative rings, Linear Algebra Appl. 397 (2005), 355-366. MR 2116468

[5] H. Chen, Rings related to stable range conditions, Series in Algebra, 11, World Scientific, Hackensack, NJ, 2011. MR 2752904

[6] J. Cui, Additive Drazin inverse preservers, Linear Algebra Appl. 426 (2007), no. 2-3, 448-453. MR 2350667.

[7] M. Dana and R. Yousefi, Formulas for the Drazin inverse of matrices with new conditions and its applications, Int. J. Appl. Comput. Math. 4 (2018), no. 1, Paper No. 4, 9 pp. MR 3736759

[8] L. Guo, J. Chen and H. Zou, Representations for the Drazin inverse of the sum of two matrices and its applications, Bull. Iranian Math. Soc. 45 (2019), no. 3, 683-699. MR 3953186

[9] L. Guo and X. Du, Representations for the Drazin inverses of $2 \times 2$ block matrices, Appl. Math. Comput. 217 (2010), no. 6, 2833-2842. MR 2733727. 
[10] B. Kuzma, Additive idempotence preservers, Linear Algebra Appl. 355 (2002), 103-117. MR 1930140

[11] S. Liu, Linear maps preserving idempotence on matrix modules over principal ideal domains, Linear Algebra Appl. 258 (1997), 219-231. MR 1444105

[12] M. Oudghiri and K. Souilah, Additive preservers of Drazin invertible operators with bounded index, Acta Math. Sin. (Engl. Ser.) 33 (2017), no. 9, 1225-1241. MR 3683708.

[13] H. Wang, J. Huang and A. Chen, The Drazin inverse of the sum of two bounded linear operators and it's applications, Filomat 31 (2017), no. 8, 2391-2402. MR 3637035

[14] K. Yan, Q. Zeng and Y. Zhu, Generalized Jacobson's lemma for Drazin inverses and its applications, Linear Multilinear Algebra 68 (2020), no. 1, 81-93. MR 4037073

[15] H. Yang and X. Liu, The Drazin inverse of the sum of two matrices and its applications, $J$. Comput. Appl. Math. 235 (2011), no. 5, 1412-1417. MR 2728075

T. P. Calci $i^{\otimes}$

Department of Mathematics, Ankara University, Ankara, Turkey

tcalci@ankara.edu.tr

H. Chen

Department of Mathematics, Hangzhou Normal University, Hangzhou, China

huanyinchen@aliyun.com

S. Halicioglu

Department of Mathematics, Ankara University, Ankara, Turkey

halici@ankara.edu.tr

G. Shile

Fuqing Branch of Fujian Normal University, Fuqing, China

gsl456@163.com

Received: December 11, 2019

Accepted: August 3, 2020 